\title{
Prediction of sperm progression in three dimensions using rapid optical imaging and dynamic mechanical modeling
}

\section{Mayssam Nassir}

Tel Aviv University

Mattan Levi

Tel Aviv University

Gili Dardikman-Yoffe

Tel Aviv University

Simcha K. Mirsky

Tel Aviv University

Natan T. Shaked ( $\nabla$ nshaked@tau.ac.il )

Tel Aviv University

\section{Research Article}

Keywords: Sperm, Interferometric computed tomography, Tomographic phase microscopy, Biomechanical modeling

Posted Date: September 28th, 2021

DOl: https://doi.org/10.21203/rs.3.rs-928380/v1

License: (1) This work is licensed under a Creative Commons Attribution 4.0 International License. Read Full License

Version of Record: A version of this preprint was published at Cells on April 13th, 2022. See the published version at https://doi.org/10.3390/cells11081319. 


\title{
Prediction of sperm progression in three dimensions using rapid optical imaging and dynamic mechanical modeling
}

Mayssam Nassir, Mattan Levi, Gili Dardikman-Yoffe, Simcha K. Mirsky, Natan T. Shaked

Department of Biomedical Engineering, Faculty of Engineering, Tel Aviv University, Tel Aviv 69978, Israel.

Authors: Mayssam Nassir, mayssam.nassir@gmail.com.

Mattan Levi, mattanlevi@gmail.com.Gili Dardikman-Yoffe, GiliDard@gmail.com.

Simcha K. Mirsky, simcha.mirsky@gmail.com.

Corresponding author: Natan T. Shaked, nshaked@tau.ac.il

\begin{abstract}
We present a multidisciplinary approach for predicting how sperm cells with various morphologies swim in three-dimensions (3D), over time scales of milliseconds to hours at spatial resolutions of less than half a micron. We created the sperm 3D geometry and built a numerical mechanical model using the experimentally acquired dynamic 3D refractive index profiles of sperm cells swimming freely in vitro as imaged by high-resolution optical diffraction tomography. By controlling parameters in the model, such as the size and shape of the sperm head and tail, we can then predict how different sperm cells, normal or abnormal, would swim in 3D, in the short or long term. We quantified various 3D structural factor effects on the sperm long-term motility. We found that some abnormal sperm cells swim faster than normal sperm cells, in contrast to the commonly-used sperm selection assumption during IVF, according to which sperm cells should mainly be chosen based on their progressive motion. We established a new tool for sperm analysis and male-infertility diagnosis, as well as new sperm selection criteria for fertility treatments.
\end{abstract}

Keywords: Sperm, Interferometric computed tomography, Tomographic phase microscopy, Biomechanical modeling 


\section{Introduction}

Human sperm cell morphology and motility are major factors in the diagnosis and prognosis of male fertility, as well as for sperm selection for in vitro fertilization (IVF), where the male sperm cells fertilize the female egg in a dish. The majority of sperm cells that reaches the fertilization sites in the female body have normal morphology according to that WHO-2010. Normal morphology also correlates with other sperm potency parameters such as hyaluronic acid binding, Zona-hamster assay and low DNA fragmentation index ${ }^{1}$. The physiological mechanisms of the female body for natural selection of sperm cells are bypassed in IVF, and it is not possible to predict which individual sperm cell would be the one that is most likely to fertilize the egg naturally and result in a healthy child. Furthermore, it is not completely clear why normal morphology sperm cells are also the good swimmers and the ones that would reach the fertilization sites in the female body. Predicting the dynamics by which a human sperm cell moves in $3 \mathrm{D}$ relative to an ambient flow and various obstacles in the female body, for long-distances, during natural fertilization, is very challenging. The biological mechanisms that connect sperm movement, morphology, contents, fertilization potential and normal pregnancy are not completely understood. Previously, microscopic analysis together with theoretical modelling were used to illustrate and interpret the swimming strategies of sperm cells under complex flow conditions ${ }^{2-6}$. Tracking of human sperm cells across large volumes by digital imaging and analysis techniques has also been demonstrated ${ }^{7-9}$. Specifically, Su, et al. ${ }^{9}$ used lens-free imaging on a chip, tracked the 3D trajectories of individual human sperm cells, and concluded that typically the sperm head moves forward swiftly along a slightly curved axis with a small lateral displacement. Dardikman-Yoffe, et al. ${ }^{10}$ presented a quantitative optical microscopy method and a set of reconstruction algorithms to provide highly detailed dynamic 3D refractive index distributions of the sperm cell during swim, including both the head with its internal organelle morphologies and the flagellum.

The sperm flagellum has a detailed internal structure, the axoneme, which is powered by dynein molecular motors distributed regularly along its length and circumference. Dynein motors produce local force, which is translated into the controlled, regular beating of the global structure

11. Considerable interest has been directed towards understanding the dynein activities by 
development of a mechanical model that includes the force generation and activation dynamics of the dynein molecular motors ${ }^{12,13}$. Sperm flagellar axoneme consists of a central pair of singlet microtubules surrounded by nine outer doublet microtubules ( $9+2$ structure) and encased by the cell membrane ${ }^{14}$. Radial links connect the central microtubule pair to each surrounding microtubule doublets, and nexin bridges connect adjacent doublets. Active and passive sliding between pairs of outer doublets by the dynein molecular motors induce shear forces and bending.

Classical models for ciliary and flagellar motion have been developed ${ }^{6,7,15-18}$. Advanced models that analyze in detail the axonemal structure and dynein activation, in order to explore and describe the internal ciliary and flagellar dynamics, were also presented ${ }^{19-22}$. Most studies developed approximate models containing mechanical parts, which represent the main organs of the sperm cell. These models only partially describe the geometrical structure of sperm cells, and consequently lead to a lack of understanding of the mechanical behavior of the sperm cell swimming. Previous models described the two-dimensional axonemal structure of planar beat patterns by two elastic rods separated by a fixed distance, linked by elastic structural elements and by active force generators, corresponding to the dynein motor proteins ${ }^{23-26}$. Specifically, a recent research modeled sperm motility in a hydrodynamic simulation to study the broad deflection-angle distribution of sperm cells in zigzag channels ${ }^{26}$. For this purpose, they implemented a minimalistic sperm model, which consisted of two successional segments connected by nodes, with each node containing four beads, which were connected by springs. Another study quantified the flagellar beats of sperm cells and determined the average time of the beating shape, as well as the first temporal Fourier mode of the beat patterns for different experimental conditions using a two-dimensional representation of the axoneme, in which there were two flexible filaments that slid relative to each other in the beat plane ${ }^{23}$. A recent study found significant differences between fertile and non-fertile men with testicular germ cell tumors, with high significant values to tail and head abnormalities ${ }^{27}$.

In the current paper, we propose a combined method that can predict how normal and pathological human sperm cells would swim in 3D based on multiple kinetic parameters describing the mechanical and physical dynamic behavior of sperm cells in 3D space. We 
previously acquired dynamic 3D profiles of freely swimming normal human sperm cells using optical diffraction tomography, a stain-free quantitative optical imaging method ${ }^{10}$. Based on the reconstructed dynamic 3D profiles of the normal cells, we develop mechanical computational sperm-cell models, for both normal and abnormal sperm morphologies. These models describe the full sperm geometry with all cell organelles (head, flagellum and midpiece), including all the internal components and structure for each organelle. We then reconstruct the mechanical behavior of each model by activating the dynein motors to describe a normal cell model behavior, and compare it to an abnormal cell model behavior by changing various parameters, such as flagellum amplitude, wavelength, beat frequency, total and axial displacements and linear and angular velocities. Using these models, we can also evaluate the 3D swimming pattern of the sperm cells for much longer periods than can be acquired optically. This approach offers an additional data that cannot be obtained using the previous methods. We provide the researcher and clinician with new information for evaluating the potential fertility of individual human sperm cells in reaching the egg, as well as provide a novel means for studying the interplay between sperm morphology and dynamics in 3D. 


\section{Results}

We distinguish between two simulation conditions: first, the case in which the sperm head is clamped (held at its tip) in a way that it cannot move forward; second, the case in which the head is free to swim in the 3D space. In the case of clamped head, we have quantified the flagellar beats of the sperm models with high spatio-temporal resolution and determined the frequency and shape amplitude for each model.

Flagellar beating of the normal sperm model

Figure 1 (a-d) show 12 frames from 3D and 2D views of the flagellar beating of the swimming normal sperm model when the head is clamped by a solid boundary. The flagellum rolls counterclockwise around its swimming axis and each flagellar point rolls in helical trajectories. The wave form of the 3D flagellar beating is described in Figure $\mathbf{1}(\mathbf{a})$, and Figure $\mathbf{1}(\mathbf{b}-\mathbf{c})$ show the projections of the 3D flagellar beat in 2D. In Figure $\mathbf{1}(\mathbf{b}-\mathbf{c})$, the flagellar wave form presents 
looping patterns with polar symmetry on both planes ( $x-y$ and $x-z)$, while in Figure $\mathbf{1}(\mathbf{d})$ the wave form presents asymmetrical behavior in the front plane $(y-z)$.
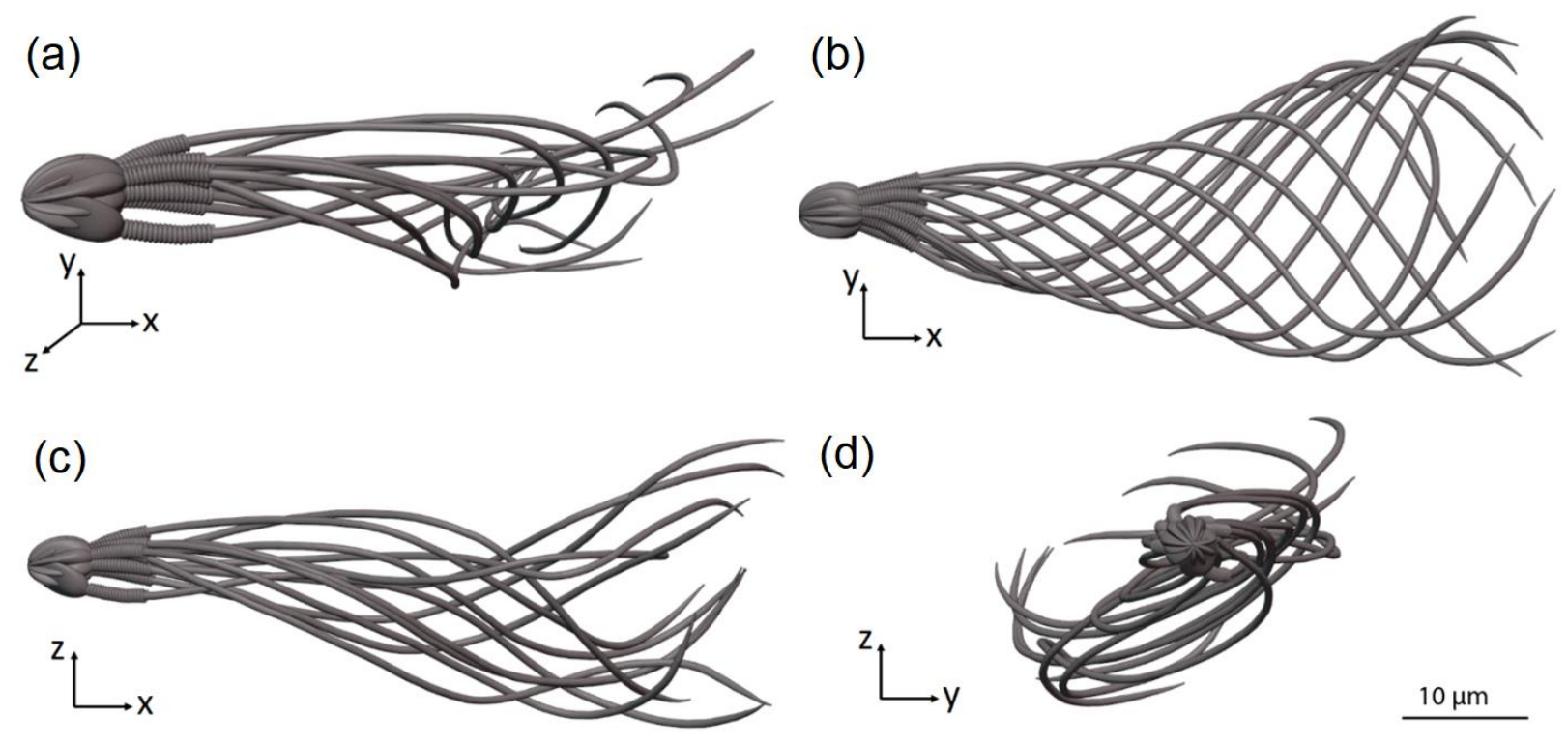

(d)

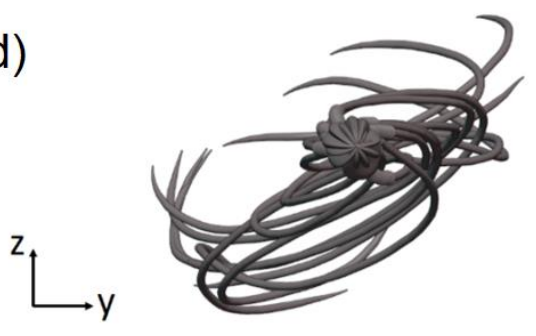

$10 \mu \mathrm{m}$

Figure 1. Flagellar beating of a normal sperm model with clamped head (held at its tip). (a) Wave form of the 3D flagellar beating. (b-d) The projections of the 3D flagellar beat in three orthogonal planes.

In the second case, we dynamically track the 3D trajectories of the sperm head centroid of each model across infinite fluid for 17 seconds, with submicron accuracy. The 3D trajectory of the normal model exhibits the most prevalent swimming pattern reported among healthy human sperm, which is called the "typical" trajectory (>90\% of cells) ${ }^{7,10}$. Figure 2 (a) shows six frames (each in a different color) from the flagellar beating of the normal model, as the sperm head changes its direction arbitrarily in 3D space over $16 \mathrm{sec}$. The path shown in Figure $\mathbf{2}(\mathbf{b}-\mathbf{e})$ indicates that the sperm head moves forward swiftly along a slightly curved axis with a small lateral displacement (approximately $4 \mu \mathrm{m}$ side-to-side) in either direction orthogonal to the flow, in agreement with earlier observations. The oscillation frequency obtained from the analysis of the waveforms shown in Figure 2 is $5.4 \mathrm{~Hz}$, and the maximum local amplitude is $7.3 \mu \mathrm{m}$; in other 
words, the head model completes 5.4 cycles (rotation of $360^{\circ}$ for each cycle) per second with internal maximum flagellum amplitude of $7.3 \mu \mathrm{m}$.

(a)

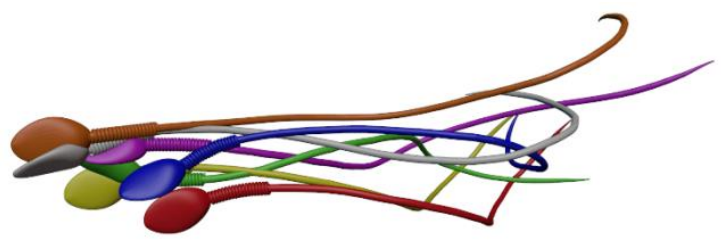

(b)

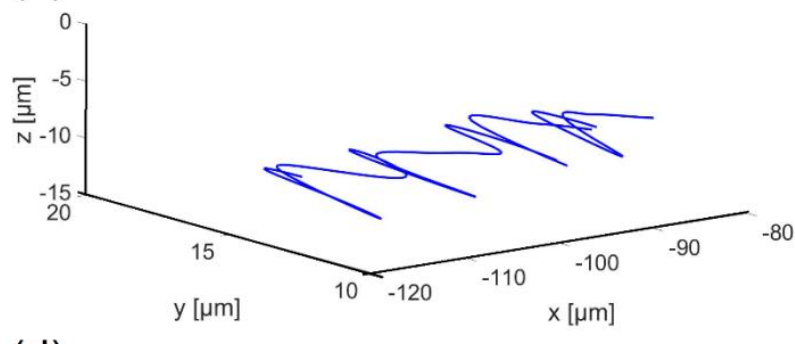

(d)

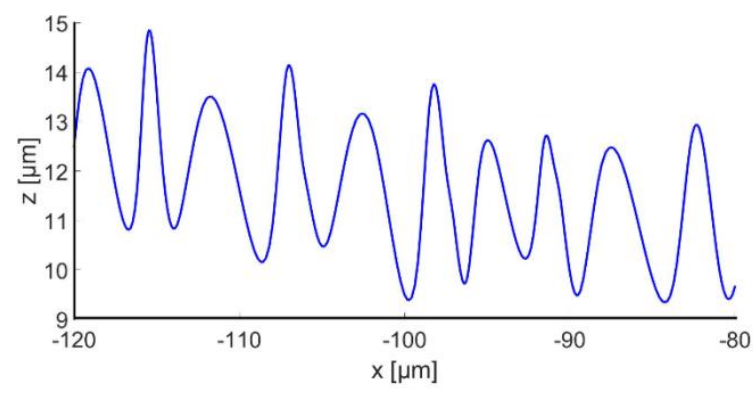

(c)

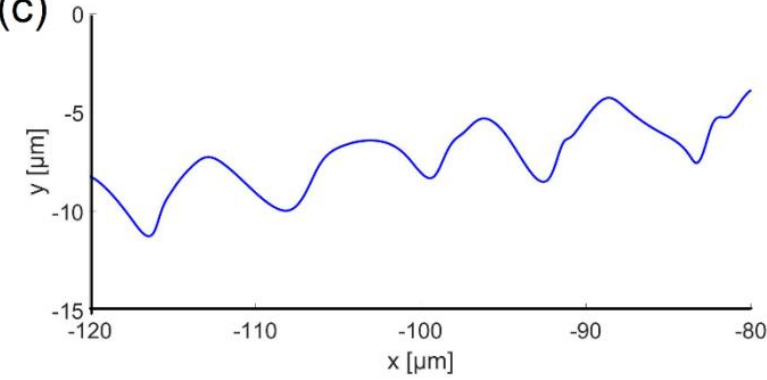

(e)

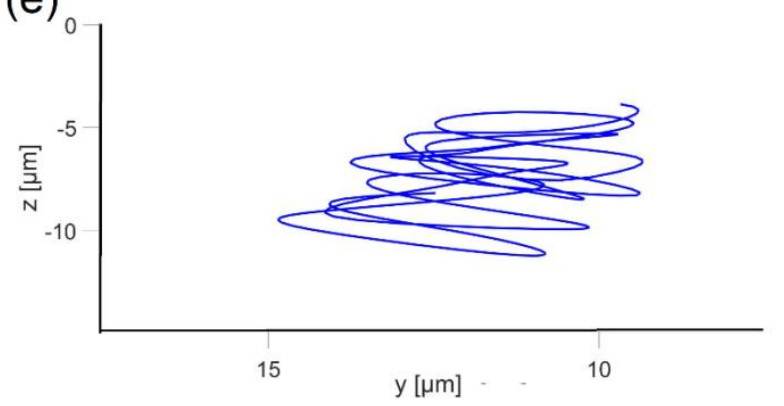

Figure 2. Flagellar beating of the swimming normal model. (a) Six frames from the 3D motion of the normal model. (b-e) Head centroid path in different planes.

\section{Normal and abnormal model swimming patterns}

During swim, we have observed that the different sperm models display a large variation in frequency, wave form, and path trend in their 3D swimming patterns (Figure 3, see Video 1). The model positions, trajectories, and displacements as a function of time are significantly different. Compared to the normal model, the abnormal sperm models exhibit different 3D swimming patterns, which show significant 3D lateral displacements, different degrees of periodicity and twisted trajectories (see Video 2 and Video 3). 


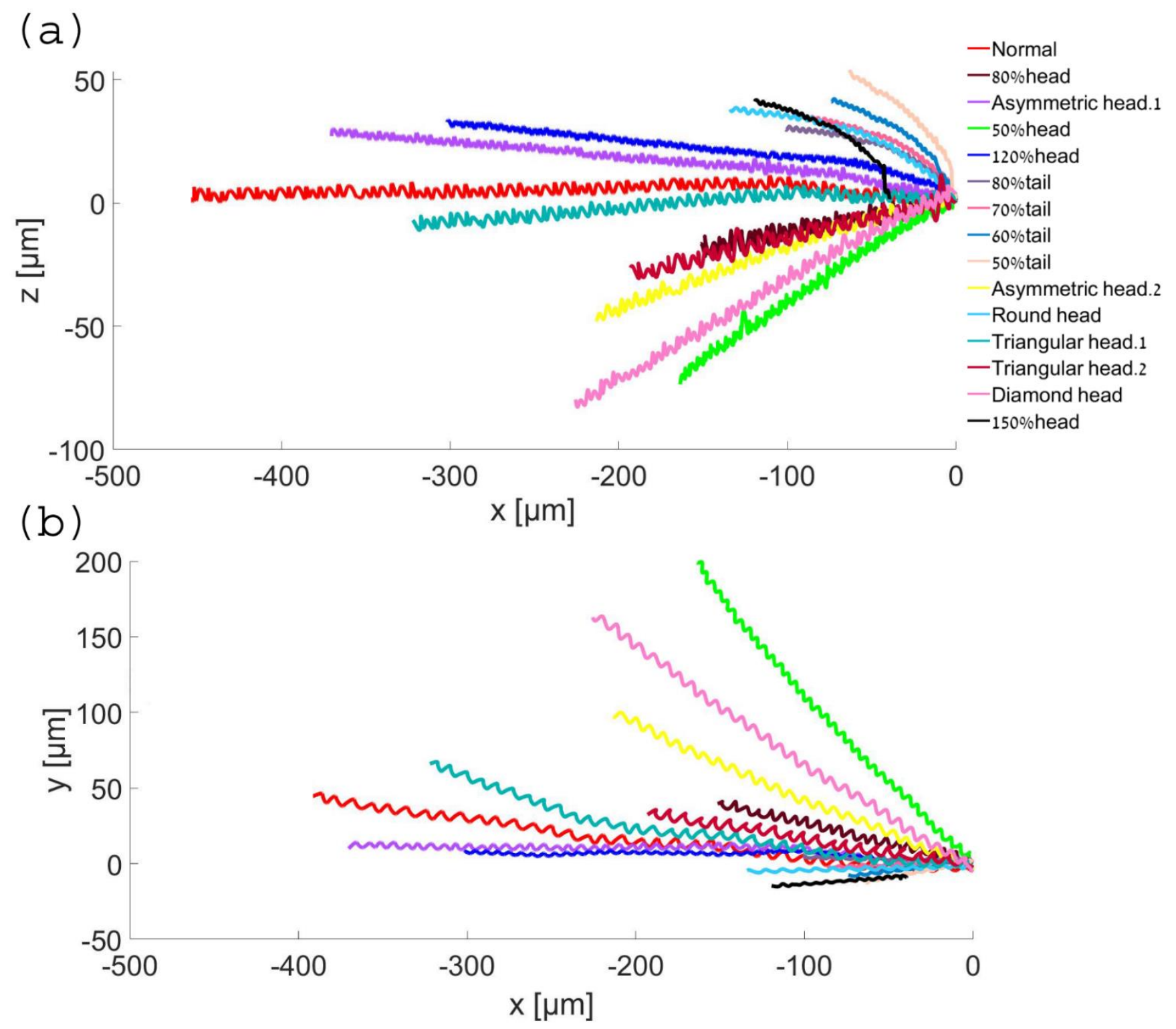

Figure 3. Normal and abnormal model swimming patterns. Two-dimensional trajectories in the forward direction (a) and in the upward direction (b).

We also quantify various physical and kinetic parameters of 3D swimming patterns and compare them to the statistical behavior of human sperms, such as flagellum amplitude, wavelength, beat frequency, total and axial displacements and linear and angular velocities (Figure 4 (a-f)). The kinetic parameters values of the normal model are equivalent to earlier reports that used different imaging techniques to track healthy human sperms 7,23,28. 
Nevertheless, the abnormal models show wide range of kinetic and physical values which prevent them from reaching the target.

\section{Kinetic parameters of $3 D$ swimming patterns}

The kinetic parameters for the models that have a short tail, large head or rounded head are smaller than those of the normal model. For the models with a defected tail, the tail does not have sufficient energy to move forward so these models swim the least distance in the forward direction $(332.5 \pm 52 \mu \mathrm{m})$. The energy of the normal cell is $E=5.36 e^{-8}$ joule, each cell with less energy will have difficulties to move itself forward. As a result, their linear and angular velocities are lower, and their motion frequency and wavelengths are decreased as well (linear velocity: $20 \pm 3 \mu \mathrm{m} / \mathrm{sec}$, angular velocity: $26.3 \pm 31 / \mathrm{sec}$, frequency: $4.2 \pm 0.5 \mathrm{~Hz}$, wavelength: $5.4 \pm 0.72 \mu \mathrm{m})$. The internal amplitude also receives the lowest values $(6.11 \pm 0.35 \mu \mathrm{m})$ for the same reason that the tail has less internal motors due to its short length, which weakens its normal beat. Even the models with a large or rounded head do not get as far from their original location, which means that their frequency, wavelength, and velocity are relatively low compared to the normal model. We can explain this by the load theory, according to which a large head increases the mass of the entire model and offloads the tail. In our case, the load is the total mass of the cell. Thus, these models swim more slowly and for smaller distances compared with the normal model.

On the other hand, the physical values of the 3D trajectories of the models with small, triangular or asymmetrical heads are significantly larger than those of the normal model. Despite the large values of rotational and linear velocity, frequency, internal amplitude and wavelength (linear velocity: $36 \pm 8.25 \mu \mathrm{m} / \mathrm{sec}$, angular velocity: $47 \pm 11.81 / \mathrm{sec}$, frequency: $7.5 \pm 1.9 \mathrm{~Hz}$, wavelength: $7 \pm 0.5 \mu \mathrm{m}$, amplitude: $10.9 \pm 3.2 \mu \mathrm{m}$ ), these defected models swim less distance in the forward direction, and with a large lateral displacement upwards (441.5 $\pm 21 \mu \mathrm{m})$, compared to the other models. These defected models are inefficient as they deviate from the straight forward swimming path, which could keep them from their goal of reaching the female egg. Moreover, a small head can indicate a deficiency of the genetic material in the nucleus, which may cause genetic problems or unsuccessful fertilization. To conclude, the mechanical behavior of the normal sperm model displays the best 3D swimming pattern for achieving the goal of reaching and fertilizing the egg. 
Lastly, we describe the mechanical behaviors of the models in terms of momentum; a vector quantity expressing the direction and intensity of the model motions to evaluate the difficulty of the body to change its velocity during free movement by:

$$
\text { (3) } P_{x, y, z}=m * v_{x, y, z}
$$

where $m$ is the model mass and $v_{x, y, z}$ is its axial velocity.

We describe the momentum in two directions, ' $x$ ' - the forward direction along the ' $x$ ' axis, and ' $y+z$ ' - the lateral direction in the ' $y-z$ ' plane (Figure 4.g). The momentum of the healthy normal model, which is calculated from its dynamic swimming parameters, has the largest value in the forward direction (561 $\mathrm{gr}^{*} \mu \mathrm{m} / \mathrm{sec}$ ), and the lowest value in the other direction (32 gr $\times \mu \mathrm{m} / \mathrm{sec})$, compared to the abnormal model values. This result indicates that the normal sperm cell applies the most energy towards moving in the forward direction, in addition to a constant velocity in the 3D space. In other words, compared to the abnormal cells, it will be more difficult to interrupt the swimming path of a normal sperm cell by collision with obstacles or with other cells. Compared with the normal model, the defect models have lower momentum values in the forward direction (436 $\left.\pm 105 \mathrm{gr}^{*} \mu \mathrm{m} / \mathrm{sec}\right)$, and larger momentum values in the lateral direction $(293.8 \pm 290 \mathrm{gr} * \mu \mathrm{m} / \mathrm{sec})$; their kinetic energy is wasted on a different direction, and not focused in the forward direction, which explains our observations that the 3D sperm swimming patterns show large lateral displacements and different degrees of periodicities. 
(a)

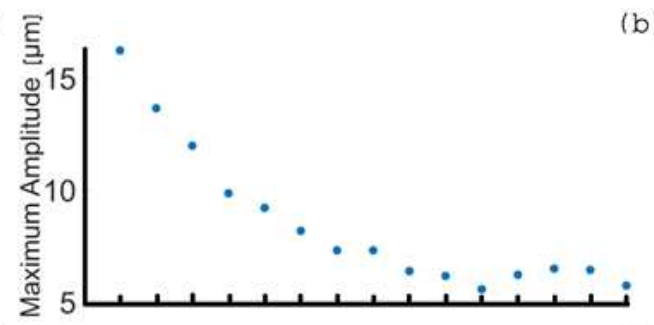

(c)

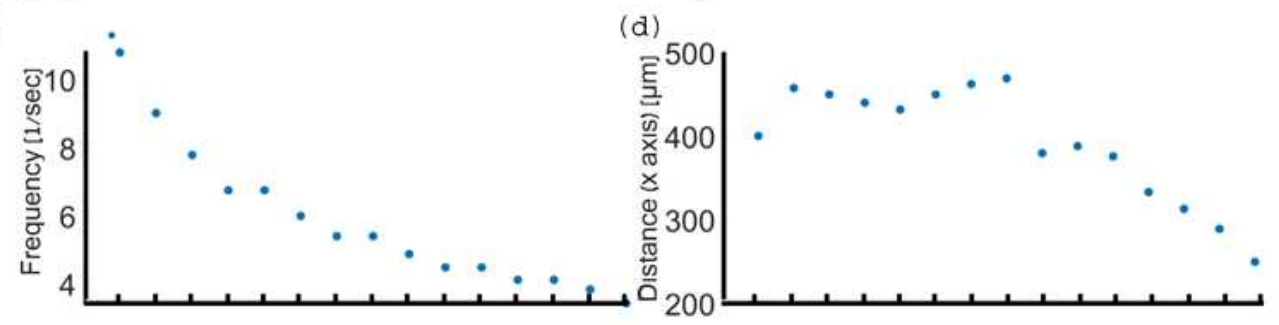

(e)

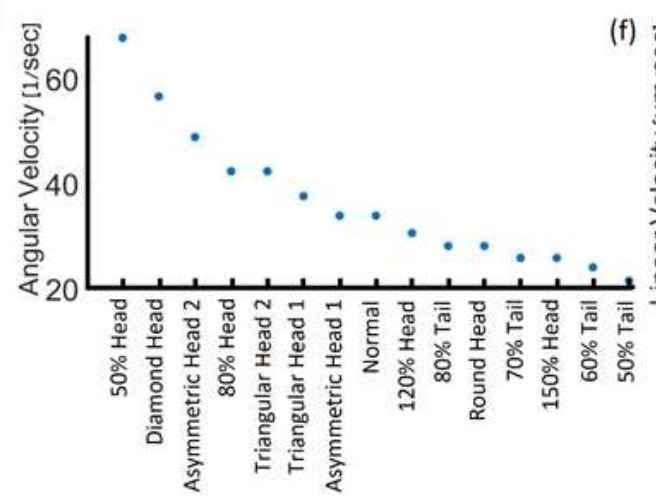

(g)

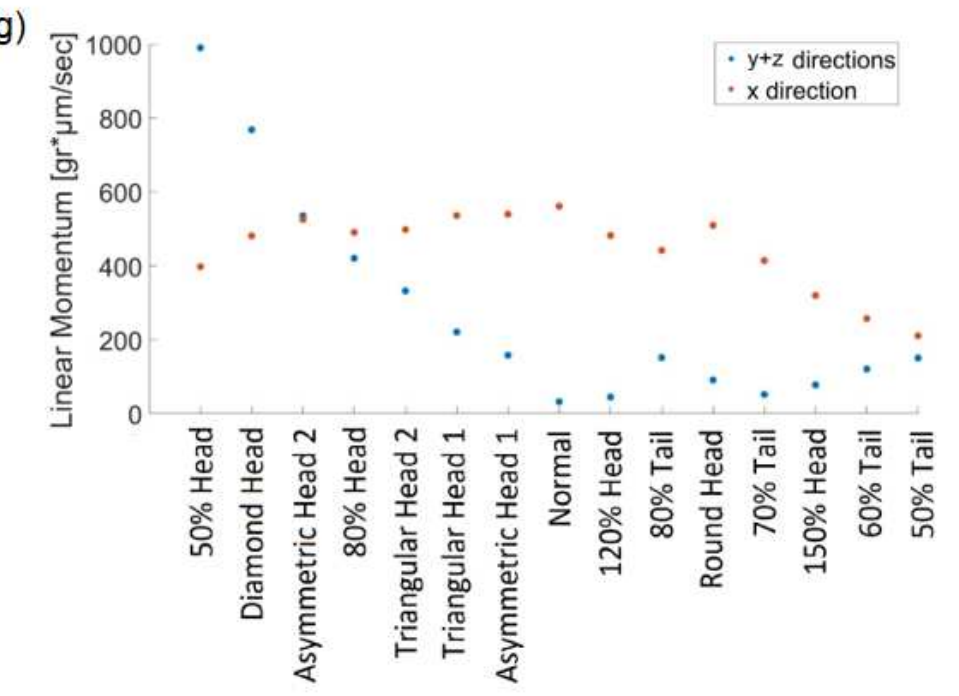

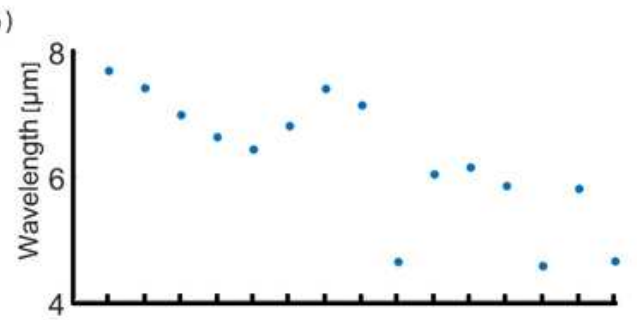

d)

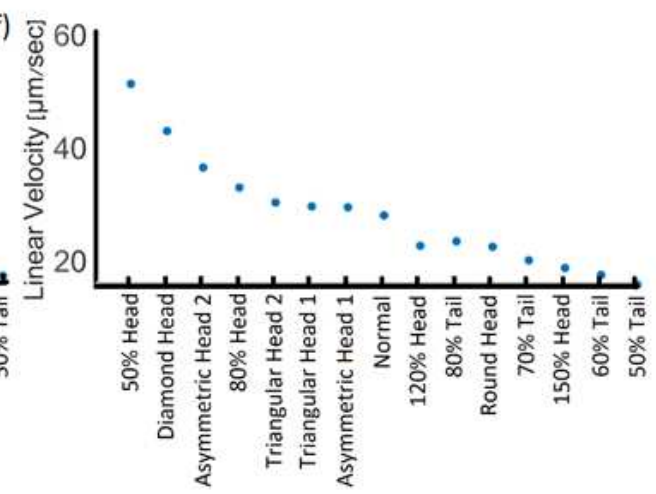


Based on the dynamic simulation described above, we performed predictions for the cell swimming paths over a longer period of time to identify global trends and the longer-term behavior of the cells. Using 3D polynomial regression, we have identified the relationship between the independent variables, which was modelled as a polynomial in 3D. Based on this, we have predicted the paths travelled over 15 minutes in 3D. Figure 5 shows the two-dimensional trajectories of the normal and abnormal cell models in the ' $x-y$ ' plane and in the ' $x-z$ ' plane. We have found that short-term trends and behavior were preserved over time, the cells keep moving forward and the gaps continue to increase over time between normal and abnormal cells. As shown earlier in the calculated path, the 3D trajectory of the normal model is the most linear along the forward direction, the ' $x$ ' axis, and this holds true for the predicted longer path here. Although the abnormal models move faster or slower in terms of 3D displacement and can cover larger distances along the ' $x$ ' axis, the normal model still has the most linear path in this direction, meaning that it is more progressive and conserves more energy. Video 4 shows the 15 cell model paths during the last $5 \mathrm{sec}$ of the $15 \mathrm{~min}$ swim. The internal oscillation, tail movement and head rotation of the sperm models are preserved over the entire 15 min duration, and the models continue to progress in the forward direction. 


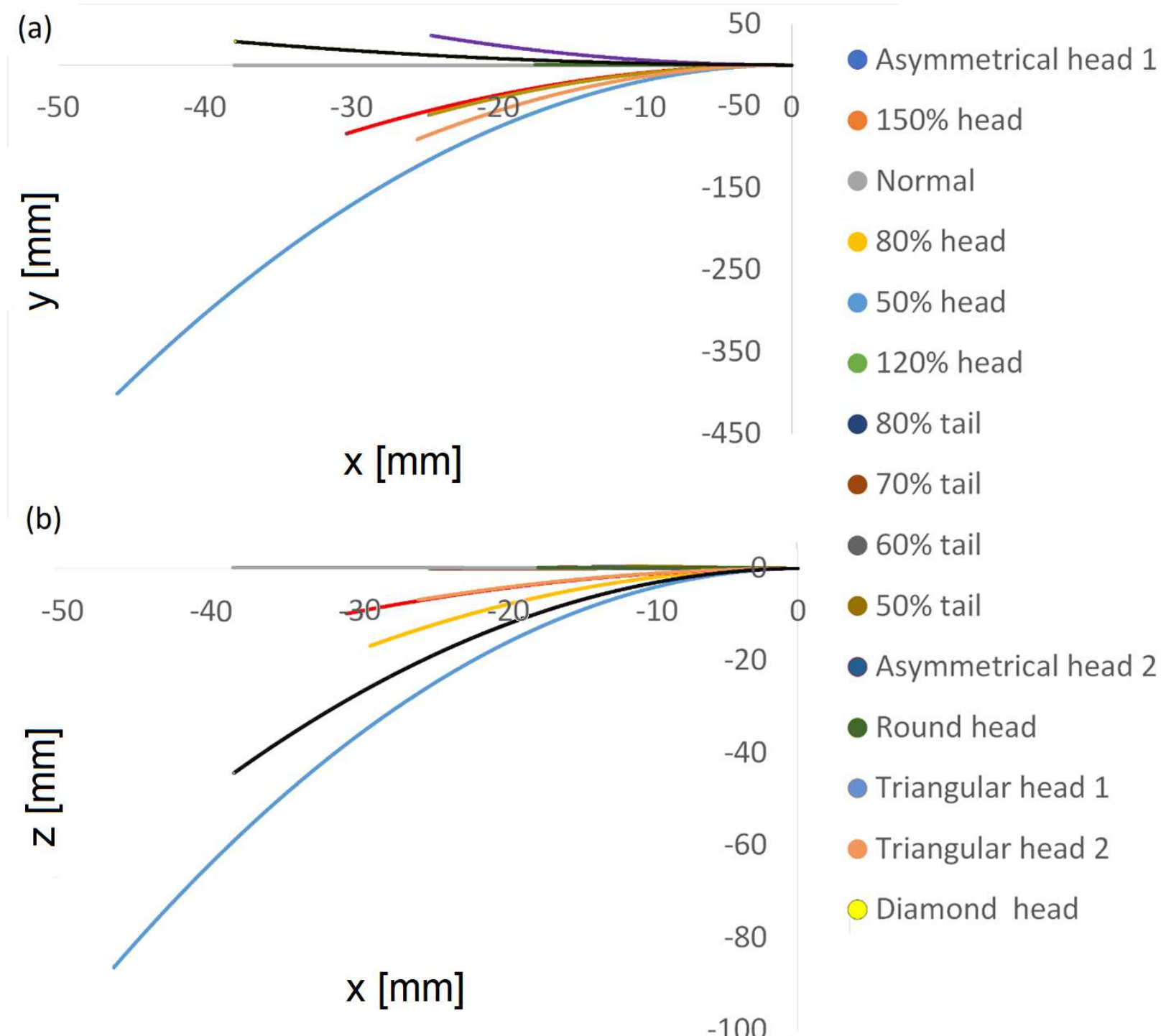

Figure 5. Normal and abnormal model swimming patterns over 15 minutes, starting on the right. Twodimensional trajectories in the ' $x-y$ ' plane (a) and in the ' $x-z$ ' plane (b). 


\section{Scoring of sperm pathologies}

Based on the results described above, we scored the various sperm cell pathologies according to their estimated success rates reaching the egg (Table 1). Cell velocity, mechanical behavior along the forward direction and the wasted energy in lateral directions were used to evaluate the success rates of the sperm reaching the egg. The models with large head have the best chances to reach the egg and the closest behavior to the normal one due to their linear path in the forward direction, nevertheless their low velocity prevents them from reaching the egg first. Thus, theoretically they would reach the egg in the second place after the normal cell. The sperm models with short tail swim slowly along the forward direction with a deviation that increases with cell shortening, which makes them less likely to reach the egg. Triangular and asymmetrical (narrow-side) heads have low chances to reach the egg because of their parabolic trajectories in the forward direction, which could keep them from their goal despite their high velocity. Small, diamond and asymmetrical (flat side) heads are the faster swimmers due to the significantly smaller mass of the entire model. However, these sperm models deviate from the straightforward swimming path and swim in the lateral direction, and have low chances to reach the egg. 
Table 1: Scoring of sperm pathologies categories according to their chances of reaching the egg.

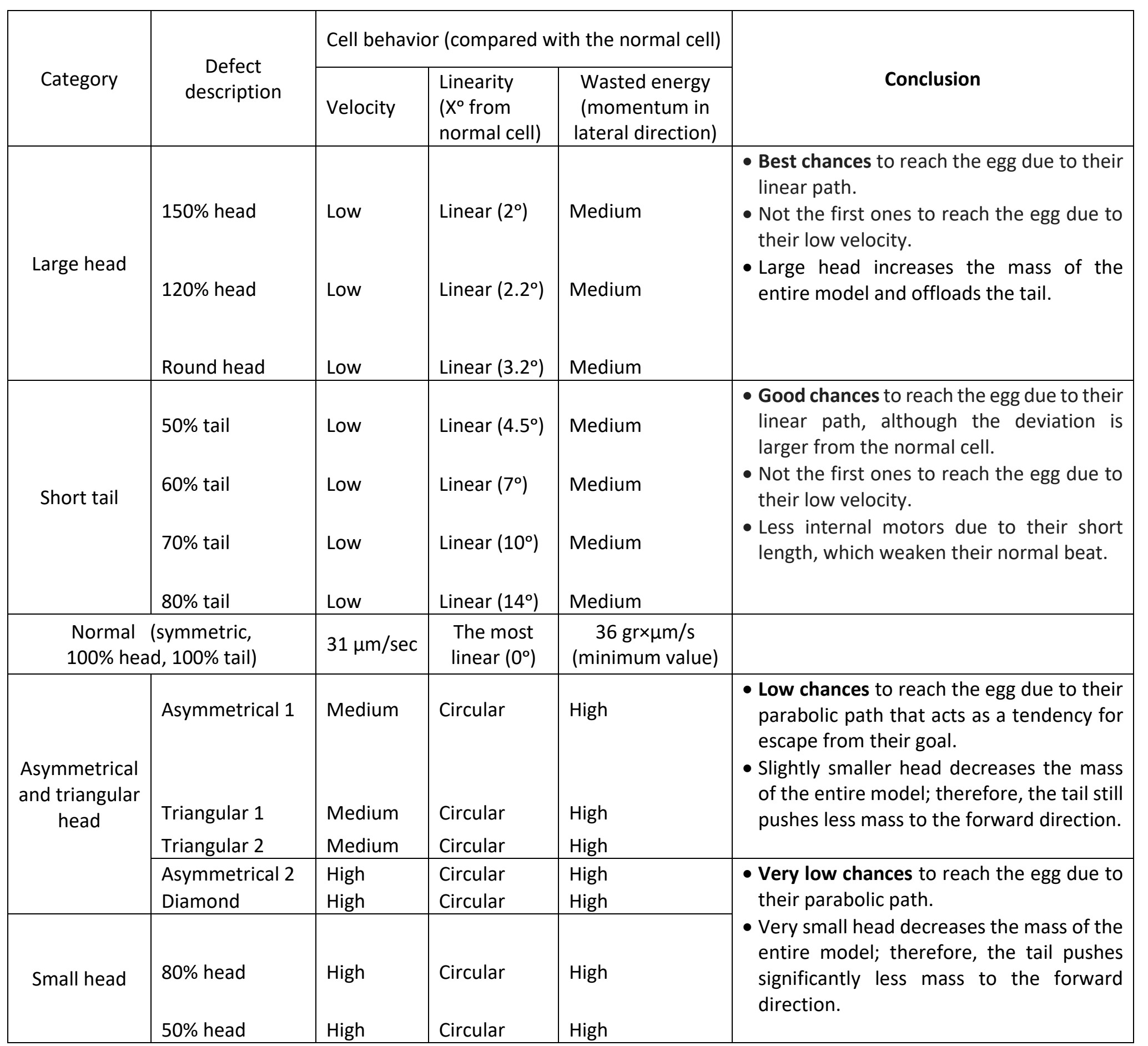




\section{Discussion}

We have described the flagellar beats of different human sperm cell models with submicron position resolution, and quantified multiple kinetic and physical parameters during free swim. We have developed 15 advanced 3D sperm cell models, presenting full sperm geometry containing the actual sperm cell organs (head, flagellum and midpiece), including internal components. The model geometry is constructed based on our previous experimental rapid optical acquisition method for individual human sperm cells during 3D movement ${ }^{10}$.

Full mechanical modeling is a key factor in understanding sperm cell motility and behavior in 3D, which directly impacts the chance of success in fertilization. A more accurate and realistic mechanical model can lead to better identification of the real movement of a swimming sperm cell, leading to improved sperm selection methods. The first model, created according to the experimental 3D video, defined a normal sperm, while the other 14 models defined sperm cells with different types of abnormalities, including known frequent defects in the sperm head and flagellum morphologies. We have performed dynamic simulations by activating dynein sliding forces using a mathematical model, characterizing the instantaneous configuration and the forward swimming of the flagellum.

From Video 3, we see that some abnormal sperm cells swim faster than normal sperm cells. This finding is very important for sperm selection in IVF, since sometimes the embryologist selects sperm cells based on their progressive motion only, whereas the sperm morphology cannot be well characterized due to using bright-field microscopy. Our findings demonstrate the necessity of imaging sperm cells in IVF with quantitative interferometric computed tomography to characterize the sperm cell morphology as well, and not only progressive motion, in order to avoid selection of pathologic sperm cells.

We have found that the kinetic parameters values of the normal model are equivalent to earlier reports that used different experimental imaging techniques to track healthy human sperm cells $1,7,10,25,27,28$. In addition, we could compare the normal sperm model with the abnormal sperm models that have shown a wide range of kinetic and physical values, which ultimately prevent them from reaching the egg. The 3D trajectory of the normal model has the 
longest path in the forward direction, as the kinetic energy is directed into forward path, without wasting energy in the lateral direction. Our results indicate that the normal model does not move the fastest in terms of 3D displacement. However, previous studies described the sperm speed only in the forward direction and showed a positive correlation between high speed and high fertilization success ${ }^{28-31}$. Our study can be used to inspect the full mechanical behavior of sperm cells in 3D space in high spatial resolution, for a much longer period than is practical experimentally.

Last to be discussed is the rotational behavior of the cells on their progression axis; this rotation grants the cells the ability to bypass obstacles smoothly and is effective resistance against losing a large amount of kinetic energy to cell collisions. The rotational motion softens the loss of energy in situations where the cell contacts with an external body. The fact that the cells have relatively negligible spiral motion relative to their progressive axis rotation emphasizes the mechanism of effectively using the kinetic energy of the cells. This behavior matches with the general evolution of the cell motion towards saving its energy for its intended use; to eventually reach the female egg and penetrate it. In the future, incorporation of environmental parameters such as flow, temperature, humidity and viscosity can advance further study of these parameters and their influence on the behavior of sperm cells. Due to the variety of viscosities and topographies in the fallopian tube, further research and advanced models are needed for predicting the ability of sperm to reach the fertilization area. We intend to expand our research to include barriers and cell-to-cell interactions, including collisions.

This study is the first in the field that predicts cell pathways and classifies cells by pathological groups, performing scoring of the success rates for each cell pathology, and evaluates the chances of reaching the egg according to the kinetic properties and behavior of the cells. Thus, this study opens the door to many additional studies in predicting sperm-cell behavior and performing informed selection processes.

To conclude, we have presented new tools for predicting 3D motion of healthy and pathological human sperm cells over short and long periods of time. Our method is based on a multidisciplinary approach, combining an ultrafast 3D optical stain-free acquisition method and mechanical modeling, and prior knowledge on sperm cell internal structure, extrapolating from 
short-term 3D optical acquisition of normal human sperm cells to short- and long-term 3D dynamic modeling of both normal and pathological cells. The presented tools are expected to provide missing links from previous studies, and may lead to changes in sperm cell analysis and selection methods. Specifically, predicting the long-term 3D motion of normal and abnormal human sperm cells and their mechanical behavior constitutes a novel capability that may help in in differentiation and selection strategies of human sperm cells for fertility characterization and treatment, as well as in cell motion research, e.g., biomimetic robotics.

\section{Materials and Methods}

We built a numerical mechanical sperm model based on previous imaging experimental results of sperm cells done by our group ${ }^{10}$. A normal-morphology sperm cell swimming freely in fluid was acquired with our dynamic interferometric computer tomography method at spatial resolution of 0.5 micron, achieving both retrieval of the 3D refractive-index profile of the sperm head, and the detailed 4D localization of the thin, highly-dynamic sperm flagellum ${ }^{10}$.

Shortly, we used the natural head rotation of the sperm cell to acquire perspective projections of the head during free swim in a dish, without using any cell staining or special sample preparations ${ }^{10}$. Each projection is acquired using our clinic-ready off-axis interferometric module connected to the exit of a simple clinical inverted microscope. This optical setup acquires the sperm-cell projection wavefronts at 2000 frames per second. All projections are then processed to reconstruct the sperm 3D refractive-index profile via optical diffraction tomography, allowing us to dynamically image the sperm cell in 3D during swimming without using cell staining. The sperm flagellum and midpiece are reconstructed via holography, which enables numerical refocusing of points in thin and sparse structures, thus enabling the reconstruction of the 3D shape of the flagellum and midpiece in a single camera exposure, providing a method to track them at high 3D spatial and temporal resolutions. Due to the high temporal resolution required, we used a fast camera with an internal buffer, which limits the practical acquisition duration to no more than one second, preventing predicting the 3D swimming of sperm for longer periods.

Based on the experimental 3D swimming of the normal-morphology sperm cell, we have developed 15 detailed dynamic 3D sperm cell models: one for a normal sperm cell and 14 for 
abnormal morphology sperm cells with different types of abnormalities. These include common defects in head and flagellum morphology. The most common sperm abnormalities, as defined in the 2010 World Health Organization (WHO-2010) guidelines ${ }^{1}$, are observed as a tapered or thin head, thin midpiece and no tail or short tail ${ }^{27}$.

The models possess full sperm geometry with all sperm cell real organs (head, flagellum and midpiece) including all internal components. The head model contains nucleus, acrosome and membrane. The midpiece (neck) is composed of centrosome and mitochondria, covered by membrane. The flagellum is constructed from nine double microtubules, a central double microtubule, radial spoke triplet, nexin links and dynein motors and covered by the membrane. We use these models to simulate active and passive sliding between pairs of outer doublets by the dynein molecular motors inducing shear forces and bending of the axoneme. By activating the dynein motors in our full mechanical model, we can mathematically describe the mechanical behavior of each model over time, including path and trend of motion, velocity, momentum, and beating pattern characteristics. These models can be used to predict how normal and pathological sperm cells would swim in 3D for durations that are much longer than can be acquired experimentally.

\section{Mechanical Model}

Normal sperm model: The model, possessing full sperm geometry including head and tail with all internal components, is based on the experimentally acquired sperm cells obtained in the previous study ${ }^{10}$. The overall length of the tail is $55 \mu \mathrm{m}$, its diameter diminishes from $1 \mu \mathrm{m}$ to 0.1 $\mu \mathrm{m}$ at the distal end, and it is covered by a membrane. The tail is structurally divided into three major parts: the midpiece of length $4 \mu \mathrm{m}$ and diameter of $1 \mu \mathrm{m}$, the principal piece of length 46 $\mu \mathrm{m}$ and diameter $0.5 \mu \mathrm{m}$, and the terminal segment of length $5 \mu \mathrm{m}$ and diameter decreasing from $0.5 \mu \mathrm{m}$ to $0.1 \mu \mathrm{m}$ at the end. Supplementary Error! Reference source not found. shows the structural comparison between the optical and geometrical models, including the head and the flagellum of the normal sperm model. 
Base on the experimental 3D swimming normal-morphology sperm cell ${ }^{10}$, we have built a 3D mechanical model of a sperm cell. The internal structure of the tail is comprised of a 3D axonemal structure of beat patterns by nine outer double semi-flexible filaments with diameters of $0.05 \mu \mathrm{m}$ and separated by a fixed distance of $0.15 \mu \mathrm{m}$, linked by stiff springs corresponding to nexin links. Radial springs, presenting radial spokes, connect each outer filament with the central double semi-flexible filaments to stabilize the structure. Dynein motor proteins, represented by $N=100$ nodes, are located along each outer double filament. The 3D axonemal structure is covered by elastic membrane, which modeled also the outer dense fibers. The midpiece contains $N=9$ doubled spiral arrays of microtubules (the axonemal structure) surrounded by mitochondria and covered by membrane (Figure 6). The head of the model is approximated by an elliptical shape, $4.2 \mu \mathrm{m}$ long and $2.85 \mu \mathrm{m}$ wide with radial symmetrical tapering reaching the tip of the head, approximated with $0.46 \mu \mathrm{m}$. The head model contains nucleus (56.75\%), acrosome (28.4\%) and membrane (14.86\%) and is connected to the tail by the midpiece (Figure 6). All models are built in Solidworks (Premium 2017 x64 Edition, USA).

For verification, we performed a structural similarity comparison between the optical and geometrical models using the structural similarity image index (SSIM), which resulted in values of $91.1 \%$ for the sperm head and $89.7 \%$ for the sperm tail. Supplementary Error! Reference source not found. andError! Reference source not found. display the local SSIM maps, including the global SSIM values. High values of global SSIM indicate high correlation and similarity between the models. 


\section{Flagellar axoneme}

(a)

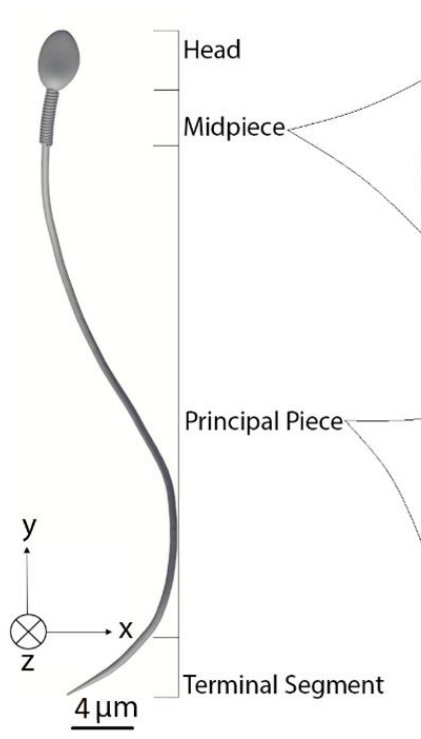

(b)

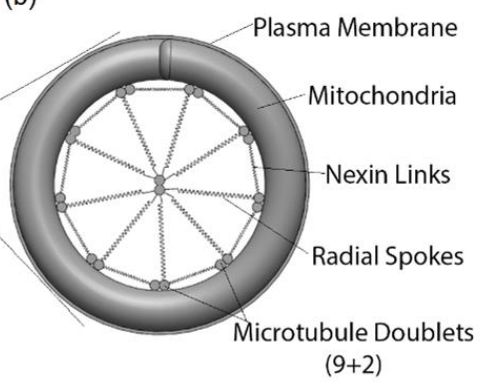

(c)

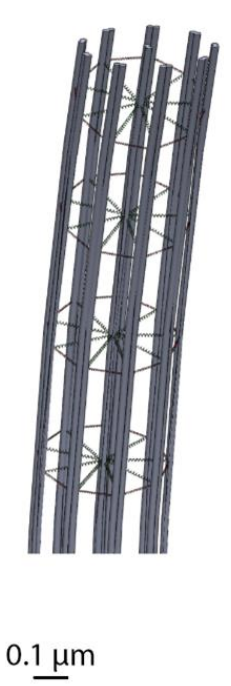

Head

(d)

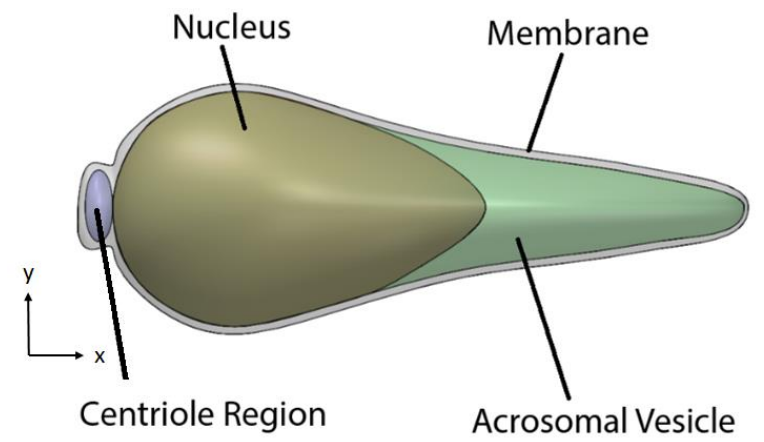

$1 \mu \mathrm{m}$ (e)

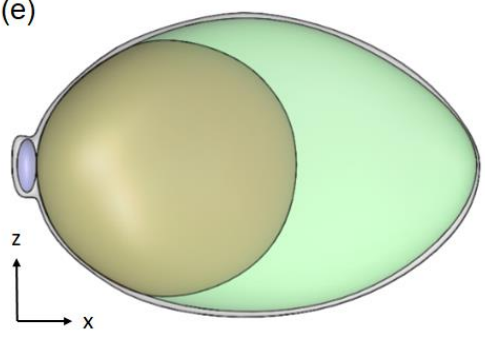

(f)

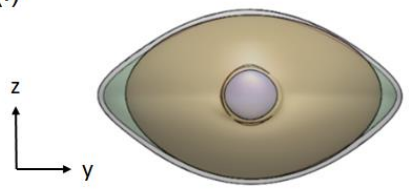

Figure 6. Sperm cell geometrical model. (a) Sperm model with all parts including head, midpiece, principal piece, and terminal segment. (b) Flagellar axoneme in cross-section: nine outer double semi-flexible filaments (microtubule doublets), linked by stiff springs (nexin links) and radial springs (radial spokes), connecting each outer filament with the central double semi-flexible filaments, covered by membrane. The midpiece contains doubled spiral arrays of mitochondria surrounded by the 9+2 axonemal structure (see (b) top). (c) 3D architecture of the flagellar structure model. 3D sperm head geometrical model: (a) Side view of the head model, containing acrosomal vesicle, nucleus, membrane and centriole region. (b,c) Top and rear views of the head model. 
Abnormal sperm models: Morphologically abnormal sperm models (Supplementary Error! Reference source not found.) are divided into three subgroups according to the defect category of the head or tail, based on real-world sperm pathologies according to the WHO-2010 guidelines.

(a) Abnormalities of head size: Four models (B-E) have abnormal head sizes (smaller or larger than normal), with tail structure and head shape as normal. The two large-headed models possess $150 \%$ (model B) and $120 \%$ (model C) of the normal head size. The two small-headed models possess $80 \%$ (model D) and 50\% (model E) of the normal head size.

(b) Abnormalities of head shape: Six models (F-K) have defects in the head shape and size, with tail structure as normal. The head defects are expressed as round head (model F), asymmetrical head on the flat side (model G), asymmetrical head on the narrow side (model $\mathrm{H}$ ), diamond head (model I), tapered head on the forward side (model J) and tapered head on the midpiece side (model K).

(c) Abnormalities of the tail length: Four models (L-O) have short tails, with head morphology as normal. Tail lengths of models $L, M, N$ and $\mathrm{O}$ are $80 \%, 70 \%, 60 \%$ and $50 \%$ of the tail length of the normal model, respectively.

\section{Beat Pattern}

The three-dimensional axoneme is divided into two groups, three and six filaments, which are located on opposing sides ${ }^{32}$. The axonemal beating is created by activating the dynein motors in two filaments groups intermittently based on the "switch-point" principle ${ }^{32-35}$. This principle specifies that the flagellar beat pattern is caused by the periodic switching of spatially restricted, asymmetrical dynein activation in the two filaments groups. In other words, axonemal bending in one direction is generated by activating the dynein motors in the same direction, while the dynein motors located along the second filament group on the opposite side are passive. Therefore, the action of dynein motors at opposite sides of the axoneme is superimposed in an antagonistic manner by switching flagellar sides relative to the bending direction. The number of the active dynein motors along each filament is determined to be $N=100$ nodes. The beating 
patterns of the different models are unaffected with larger numbers of nodes but influence the simulation runtime and memory. Low simulation resolutions are obtained with less than 100 nodes of dynein motors.

We have simulated the dynein sliding forces by a mathematical model based on position changes. We describe the shape of a filament by its curvature, $r(s, t)$, where $s$ is the dynein component. Each elastic filament can bend along the normal $(\hat{l})$ and radial $(\hat{\jmath})$ directions, and twisted along the tangent direction $(\hat{k})^{36}$. The change in the 3D positions along the curve of each filament is given by the Frenet equations:
(1) $\frac{d T}{d s}=\kappa N$,
(2) $\frac{d N}{d s}=\tau B-\kappa T$,
(3) $\frac{d B}{d s}=-\tau N$,

where $\kappa$ is the curvature, and $\tau$ is the torsion of the curve. The tangent $(T)$, normal $(N)$, and binormal $(B=T \times N)$ unit vectors are the Frenet-Serret frame. Hence, the curvature $\kappa$ and torsion $\tau$ of the curve are described by:

$$
\begin{aligned}
& \text { (4) } \kappa=\left|\frac{d T(s)}{d s}\right|, \\
& \text { (5) } \tau=\frac{1}{\kappa} \frac{d T}{d s} \frac{d B}{d s} .
\end{aligned}
$$

The following equations define the twist degree by the intrinsic twist $(\varphi)$ with respect to the Frenet frame:

$$
\begin{aligned}
& \text { (6) } \quad N(s)=\cos \varphi i-\sin \varphi j, \\
& \text { (7) } \quad B(s)=\sin \varphi i+\cos \varphi j, \\
& \text { (8) } \quad T(s)=k(s) .
\end{aligned}
$$

The change of the coordinate axis as function of time is defined by the angular velocity vector of the Frenet frame of the curve $(\omega)$. This angular velocity in each segment is the combination of the rotations of each of the three Frenet vectors: $\omega=\omega_{T}+\omega_{N}+\omega_{B}$. Infinitesimally small changes of the orientation related to rotations around the three axes are: 
(9) $\quad \partial_{s}\left(\begin{array}{c}i(s) \\ j(s) \\ k(s)\end{array}\right)=\left(\begin{array}{ccc}0 & \omega_{T}(s) & -\omega_{B}(s) \\ -\omega_{T}(s) & 0 & \omega_{N}(s) \\ \omega_{B}(s) & -\omega_{N}(s) & 0\end{array}\right)\left(\begin{array}{c}i(s) \\ j(s) \\ k(s)\end{array}\right)$.

Following the above equations, the curvature $(\kappa)$ and torsion $(\tau)$ of the curve are given by:

$$
\begin{aligned}
& \text { (10) } \quad \kappa=\left|\frac{d T(s)}{d s}\right|=\sqrt{\left(\omega_{N}\right)^{2}+\left(\omega_{B}\right)^{2}}, \\
& \text { (11) } \tau=\frac{1}{\kappa} \frac{d T}{d s} \frac{d B}{d s}=\omega_{T}-\varphi .
\end{aligned}
$$

By inverting equations $(10(11)$, and using the intrinsic twist $(\varphi)$, the local curvatures in each dynein node along the active filament are:

$$
\begin{aligned}
& \omega_{N}(s, t)=\kappa(s, t) \sin \varphi(s, t) \\
& \omega_{B}(s, t)=\kappa(s, t) \cos \varphi(s, t) \\
& \omega_{T}(s, t)=\tau(s, t)+\frac{d \varphi}{d s}(s, t)
\end{aligned}
$$

The curvature $\kappa(s, t)$ of the filament is given by ${ }^{37}$ :

$$
\text { (15) } \kappa(s, t)=s^{2} \sin \left(\frac{\pi}{3}\right)^{-1} * \delta_{N}(s, t)
$$

where

$$
\text { (16) } \delta_{N}(s, t)=A \sin \left(\frac{2 \pi}{\lambda} s-\omega t\right) \text {. }
$$

The amplitude $\left(A=0.63 L_{\text {flagellum_length }}\right)$, the wavelength $\left(\lambda=0.05 r_{\text {head_radius }}\right)$, and the frequency $(\omega=0.05 \mathrm{~Hz})$ are the mechanical properties of the stiff springs ${ }^{2623}$. The equations in the dynein motors along the inactive filaments are $\delta_{N}=\kappa(s, t)=\omega_{N, B, T}=0$.

\section{Dynamic Simulation}

To perform dynamic simulations, we have meshed the 3D geometrical sperm models. We have used the generation and processing toolbox by Solidworks to create a 3D tetrahedral element mesh for this purpose. The mesh densities of the different models vary between the cells. This variability can affect the simulations runtime, but do not change the outcome measures (less than $3 \%$ ). The models are assumed to be hyper-elastic uncompressible materials with NeoHookean constitutive model. We first analyzed the beating patterns of the sperm models when 
their heads clamped to fixed surface. The head is fixed for any progress, and free for 3D rotations. Secondly, we studied the sperm swimming inside an infinite Newtonian fluid when the head is free to swim. This Newtonian fluid is considered as a saline water with viscosity of $(1.02 \mathrm{mPa} \times$ s) at room temperature $\left(25^{\circ} \mathrm{C}\right)$. We have assumed that there was no cell-surface interaction, that is, no slip at the sidewalls, since the changes of the axonemal shape were caused by the internal force generators and unaffected by the hydrodynamics. In addition, since the simulation still ensures low Reynold's hydrodynamics, the qualitative description still holds. The dynamic simulations are performed using Blender 2.91 and Python 3.3 SW (3D modelling and rendering package).

Video 5 displays a validation of the flagellar swimming pattern obtained from the dynamic simulation. We verify that the results of the dynamic simulation describe virtually the actual swimming pattern of sperm cell, experimentally acquired in our previous study ${ }^{10}$.

\section{Outcome measurements:}

The beating patterns of the different models were analyzed for position maps, calculated by Blender 2.91 Python 3.3 SW (3D modelling and rendering package). The localized displacements that developed in the dynein nodes, heads, and the flagellar axoneme of the normal and pathological models were determined as a function of the time. Outcome measures included: (1) flagellar beatings of the normal and pathological models with clamped heads. (2) The threedimensional trajectories of the sperm head centroid of the normal and pathological models, across infinite fluid for 17 seconds. (3) The kinetic and physical parameters of the threedimensional swimming patterns, such as:

a. Distance ( $x$ axis): the axial displacement of the head centroid path in the forward direction.

b. Flagellum amplitude, calculated based on snapshots of the beating pattern which projected on the forward plane ' $x-y$ '.

c. Frequency $f=1 / T$, where $T$ is the time taken to complete one cycle of the beating pattern. 
d. Linear velocity $v=x / t$, where $x$ is the distance ( $\mathrm{x}$ axis) in the forward direction as a function of time $t$.

e. Wavelength $\lambda=v / f$, where $v$ is the linear velocity and $f$ is the frequency.

f. Angular velocity $w=v / r$, where $v$ is the linear velocity, and $r$ is the radius of the motion in the lateral plane ' $z-y$ '.

g. Momentum $P_{x, y, z}=m * v_{x, y, z}$, where $m$ is the model mass and $v_{x, y, z}$ is its axial velocity. (4) Prediction for the normal and pathological models swimming patterns over 15 minutes, using 3D polynomial regression. (5) Scoring the various sperm cell pathologies according to their estimated success rates reaching the egg. 


\section{Bibliography}

1. Organization, W. H. WHO laboratory manual for the examination and processing of human semen, fifth edition. (2010).

2. Yundt, A. P., Shack, W. J. \& Lardner, T. J. Applicability of hydrodynamic analyses of spermatozoan motion. J. Exp. Biol. 62, 27-41 (1975).

3. Ohmuro, J. \& Ishijima, S. Hyperactivation is the mode conversion from constantcurvature beating to constant-frequency beating under a constant rate of microtubule sliding. Mol. Reprod. Dev. 73, 1412-1421 (2006).

4. Daloglu, M. U. et al. Label-free 3D computational imaging of spermatozoon locomotion, head spin and flagellum beating over a large volume. Light. Sci. Appl. 7, 17121 (2018).

5. Gillies, E. A., Cannon, R. M., Green, R. B. \& Pacey, A. A. Hydrodynamic propulsion of human sperm. J. Fluid Mech. 625, 445-474 (2009).

6. Poola, P. K., Jayaraman, V., Chaithanya, K., Rao, D. \& John, R. Quantitative label-free technique for morphological evaluation of human sperm. OSA Contin. 1, 1215-1225 (2018).

7. Su, T.-W., Xue, L. \& Ozcan, A. High-throughput lensfree 3D tracking of human sperms reveals rare statistics of helical trajectories. Proc. Natl. Acad. Sci. 109, 16018 LP - 16022 (2012).

8. Bukatin, A., Kukhtevich, I., Stoop, N., Dunkel, J. \& Kantsler, V. Bimodal rheotactic behavior reflects flagellar beat asymmetry in human sperm cells. Proc. Natl. Acad. Sci. 112, 15904 LP - 15909 (2015).

9. Kantsler, V., Dunkel, J., Blayney, M. \& Goldstein, R. E. Rheotaxis facilitates upstream navigation of mammalian sperm cells. Elife 3, e02403-e02403 (2014).

10. Dardikman-Yoffe, G., Mirsky, S. K., Barnea, I. \& Shaked, N. T. High-resolution 4-D acquisition of freely swimming human sperm cells without staining. Sci. Adv. 6, eaay7619 
(2020).

11. Fauci, L. J. \& Dillon, R. Biofluidmechanics of reproduction. Annu. Rev. Fluid Mech. 38, 371-394 (2005).

12. Gaffney, E. A., Gadêlha, H., Smith, D. J., Blake, J. R. \& Kirkman-Brown, J. C. Mammalian sperm motility: observation and theory. Annu. Rev. Fluid Mech. 43, 501-528 (2011).

13. Camalet, E. \& Jülicher, F. Generic aspects of axonemal beating. New J. Phys. 2, 1-2423 (2000).

14. Lindemann, C. B. \& Lesich, K. A. Flagellar and ciliary beating: the proven and the possible. J. Cell Sci. 123, 519-528 (2010).

15. Brokaw, C. Computer simulation of flagellar movement IX. oscillation and symmetry breaking in a model for short flagella and nodal cilia. Cell Motil. Cytoskeleton 60, 35-47 (2005).

16. Hines, M. \& Blum, J. J. Bend propagation in flagella. I. derivation of equations of motion and their simulation. Biophys. J. 23, 41-57 (1978).

17. Abakuks, A. The Dynamics of Cellular Motility. Biometrics 50, 589-590 (2021).

18. Shingyoji, C., Higuchi, H., Yoshimura, M., Katayama, E. \& Yanagida, T. Dynein arms are oscillating force generators. Nature 393, 711-714 (1998).

19. Dillon, R. \& Fauci, L. An integrative model of internal axoneme mechanics and external fluid dynamics in ciliary beating. J. Theor. Biol. 207, 415-430 (2001).

20. Dillon, R., Fauci, L. \& Omoto, C. Mathematical modeling of axoneme mechanics and fluid dynamics in ciliary and sperm motility. Copyright 10, 745-757 (2003).

21. Mitchell, D. R. Orientation of the central pair complex during flagellar bend formation in chlamydomonas. Cell Motil. 56, 120-129 (2003).

22. Gueron, S. \& Levit-Gurevich, K. A three-dimensional model for ciliary motion based on the internal 9+2 structure. Proceedings. Biol. Sci. 268, 599-607 (2001).

23. Riedel-Kruse, I. H. \& Hilfinger, A. How molecular motors shape the flagellar beat. HFSP J. 1, 192-208 (2007).

24. Hilfinger, A., Chattopadhyay, A. \& Jülicher, F. Nonlinear dynamics of cilia and flagella. Phys. Rev. E. Stat. Nonlin. Soft Matter Phys. 79, 51918 (2009). 
25. Sartori, P., Geyer, V. F., Scholich, A., Jülicher, F. \& Howard, J. Dynamic curvature regulation accounts for the symmetric and asymmetric beats of Chlamydomonas flagella. Elife 5, (2016).

26. Rode, S., Elgeti, J. \& Gompper, G. Sperm motility in modulated microchannels. New J. Phys. 21, 13016 (2019).

27. Chenoweth, P. J. Genetic sperm defects. Theriogenology 64, 457-468 (2005).

28. Holt, W. V, Moore, H. D. M. \& Hillier, S. G. Computer-assisted measurement of sperm swimming speed in human semen: correlation of results with in vitro fertilization assays. Fertil. Steril. 44, 112-119 (1985).

29. Ren, S.-S., Sun, G.-H., Ku, C.-H., Chen, D.-C. \& Wu, G.-J. Comparison of four methods for sperm preparation for IUI. Arch. Androl. 50, 139-143 (2004).

30. Liu, J., Leung, C., Lu, Z. \& Sun, Y. Quantitative analysis of locomotive behavior of human sperm head and tail. IEEE Trans. Biomed. Eng. 60, 390-396 (2013).

31. Matsuura, K. et al. Screening of sperm velocity by fluid mechanical characteristics of a cyclo-olefin polymer microfluidic sperm-sorting device. Reprod. Biomed. Online 24, 109115 (2012).

32. Satir, P. \& Matsuoka, T. Splitting the ciliary axoneme: implications for a 'switch-point' model of dynein arm activity in ciliary motion. Cell Motil. Cytoskeleton 14, 345-358 (1989).

33. Ishibashi, K., Sakakibara, H. \& Oiwa, K. Force-Generating Mechanism of Axonemal Dynein in Solo and Ensemble. International Journal of Molecular Sciences vol. 21 (2020).

34. Lin, J. \& Nicastro, D. Asymmetric distribution and spatial switching of dynein activity generates ciliary motility. Science (80-. ). 360, eaar1968 (2018).

35. Satir, P., Heuser, T. \& Sale, W. S. A Structural Basis for How Motile Cilia Beat. Bioscience 64, 1073-1083 (2014).

36. A. Hilfinger. Dynamics of Cilia and Flagella. (2005).

37. Brokaw, C. J. Bend propagation by a sliding filament model for flagella. J. Exp. Biol. 55, 289 LP - 304 (1971). 


\section{Acknowledgment}

Author Contributions: MN designed the research, built the mechanical models, analyzed data, and wrote the paper. ML designed the research and contributed to the paper writing. GDY analyzed the optical experiment data. SKM performed the optical experiments and contributed to the paper writing, NTS conceived the idea, designed the research, wrote the paper, and supervised the research.

Competing interests: No potential conflict of interest was reported by the authors. 


\section{Supplementary Files}

This is a list of supplementary files associated with this preprint. Click to download.

- supplementaryinformation.zip 